\title{
ANÁLISIS DEL SECTOR AGROPECUARIO COLOMBIANO EN EL CONTEXTO DE LA PROBLEMÁTICA SOCIAL LATINOAMERICANA
}

Guillermo Peñaranda Cáceres*

* Docente del departamento de Agrarias Facultad de Ciencias Económicas y Empresariales Universidad de Pamplona. Pamplona Colombia. E-mail: german1952@mail.ru 


\section{ANÁLISIS DEL SECTOR AGROPECUARIO COLOMBIANO EN EL CONTEXTO DE LA PROBLEMÁTICA SOCIAL LATINOAMERICANA}

\begin{abstract}
RESUMEN
Formar empresarios del campo es el nuevo reto al que se encuentran enfrentados los campesinos. Se hace necesaria una transformación radical del campo, de la mentalidad del campesino y esta debe ocurrir especialmente desde la escuela primaria, las escuelas agropecuarias y las universidades. Formar técnicos agropecuarios con una mentalidad más dinamizadora de los procesos de producción agropecuaria, llevando al campo nuevas tecnologías, procesos mas eficientes, aplicando agricultura orgánica para ir cerrando la brecha de la agricultura tradicional convencional, para producir con tecnologías mas limpias, y de esta forma dinamizar el proceso de producción agrícola para producir alimentos mas sanos y saludables a la población colombiana.
\end{abstract}

Palabras Claves: Procesos productivos, rentabilidad agropecuaria, Educación empresarial para el campo, diversificación agropecuaria, agricultura autogestionaria.

\begin{abstract}
Farmers now face the new challenge of becoming entrepreneurs. This requires a radical transformation of the country side and of peasant mentality which must begin at primary school, through to agricultural college and universities. We need to form agricultural technicians with and innovativ mentalit that will make agricultural processes more efficient and using organic agriculture to close the gap with traditional agricultural practices. The result will be cleaner technologies which will significantly improve agricultural production and produce healthier food for the Colombian people.
\end{abstract}

Key Words: Productive process, Agricultural cost-effectiveness, Business studies related to the countryside, Agricultural diversification, Self-managed agriculture.

\section{Face IssN 1794-9920}

Recepción: Abril de 2008

Revisión: Mayo de 2008

Aceptación: Mayo de 2008 


\section{INTRODUCCIÓN}

Actualmente los países de América Latina y el Caribe con la globalización están enfrentados a la necesidad de aumentar rápidamente la producción agropecuaria; mejorar la calidad y reducir los costos de los productos alimenticios, para que éstos sean compatibles con el bajo poder adquisitivo de la mayoría de los consumidores nacionales y además ser competitivos en los mercados internacionales; mejorar los ingresos de los agricultores; generar empleos y ofrecer atrayentes condiciones de vida para las familias rurales en su propio medio, y con ello disminuir el éxodo rural y sus dramáticas consecuencias como esta sucediendo actualmente en nuestro país, y en muchos países de América Latina.

Para lograr lo anterior, es absolutamente indispensable promover la modernización del sector agropecuario y la tecnificación de la agricultura, volviéndola más productiva, eficiente, rentable y competitiva. Si no se moderniza el sector agropecuario y no se tecnifica la agricultura, sencillamente no habrá mayor productividad, ni eficiencia, y consecuentemente no podrá haber rentabilidad ni competitividad; esta condicionalidad es tan evidente, que está fuera de discusión. Además, es preciso llevar a cabo esta modernización en forma equitativa, es decir, hacerlo de manera tal que todos los agricultores tengan reales oportunidades de beneficiarse de estos avances tecnológicos; porque sólo así, todos ellos podrán hacer un aporte al desarrollo nacional con la eficiencia que exigen los tiempos modernos y sólo así habrá equidad y democracia real.

Lograr dicha equidad es prácticamente imposible si se sigue adoptando el modelo de desarrollo agropecuario convencional, si no se implementa un desarrollo mas equitativo con modelo de desarrollo endógeno. Con el modelo convencional los agricultores dependen excesivamente de factores externos a sus predios, tales como: las decisiones del gobierno, muchas veces inadecuadas e inoportunas; el crédito y otros servicios del Estado, generalmente insuficientes e ineficientes; las semillas y sementales de alto potencial genético; los insumos y los equipos modernos; los subsidios y las garantías oficiales de precios y de comercialización; es imposible por la sencilla razón de que, según informes de la FAO, más del noventa por ciento de los agricultores de América Latina y el Caribe no tienen acceso, en forma completa, permanente y eficiente a dichos factores externos; es decir, "los enfermos no pueden comprar las medicinas que el médico les recomienda". En tales circunstancias y de seguir adoptando dicho modelo convencional, no puede haber crecimiento agropecuario con equidad, debido a que no existen recursos en cantidad suficiente para que todos los agricultores puedan acceder a los factores externos recién mencionados (ni los productores los poseen, ni los gobiernos pueden proporcionárselos).

El ignorar o subestimar esta verdad tan patente, ha sido la principal causa por la cual han fracasado la mayoría de los muchos proyectos lanzados en los países de la Región con el 
fin de promover el desarrollo del sector agropecuario. Es necesario romper esta contradicción entre lo que se desea y lo que realmente se puede hacer. Teóricamente, existirían dos alternativas para lograrlo. La primera sería incrementar el volumen de los recursos destinados al desarrollo del sector agropecuario, de modo de poder ofrecer todos los factores antes mencionados a la totalidad de los agricultores; hipótesis ésta absolutamente insostenible y fuera de contexto dentro de los ajustes estructurales y de la actual situación de crisis de los países. La segunda hipótesis -ésta sí más realista- sería adoptar un modelo alternativo de desarrollo agropecuario, que posibilite a los agricultores protagonizar un desarrollo más endógeno, más autogestionario y más autogenerado, para que ellos se vuelvan menos dependientes de los factores escasos antes mencionados, es decir: de las decisiones del gobierno, de los servicios del Estado y de los recursos externos a sus fincas y comunidades.

Ello significa modernizar la agricultura a partir del uso racional de los recursos internos que poseen los agricultores y potenciarlos con la correcta introducción de tecnologías de bajo costo, adecuadas a las adversidades físico productivas y a la escasez de recursos de capital; porque son éstas las circunstancias reales que caracterizan a la gran mayoría de los productores agropecuarios de esta Región (América Latina y el Caribe). La necesidad de promover un desarrollo menos dependiente de factores externos se ha hecho más evidente y urgente, debido a la orientación neoliberal en la cual los gobiernos tienden a disminuir aún más sus atribuciones, su aparato de apoyo al agro y los ya escasos recursos que destinan al desarrollo del medio rural y muchas, por no decir la gran mayoría de veces, no se llega al campo ni siquiera con programas de electrificación rural, saneamiento básico (agua potable y demás anexos), educación para el campo, vías de penetración y asistencia técnica especializada para cada región, que permita a los agricultores ser mas competitivos y que no sigan nadando en la pobreza extrema.

El ejemplo mas patente actualmente en la crisis colombiana radica en que se acabaron prácticamente los servicios de extensión agropecuaria por parte del estado, como el caso de la caja Agraria, las UMATAS y de otros entes bancarios, así como también del ICA y Federaciones de gremios agrícolas. A esto hay que agregarle la situación de orden público a la que se abandonaron poblaciones enteras, dejándolas en el caos y abandono, entregándolas prácticamente a las fuerzas insurgentes de uno y otro bando que azotaron la geografía colombiana, causando un éxodo masivo a las grandes ciudades del país.

Es de imperiosa necesidad comenzar proyectos de retorno al campo. Iniciar con una nueva experiencia sería prepararlos para una nueva vida que implicaría otro modelo de desarrollo y este nuevo modelo de desarrollo sería más autogestionario (el agricultor como agente de su autodesarrollo) y más endógeno (basado en los recursos que los agricultores realmente poseen), exige la difusión de nuevas tecnologías agropecuarias, la capacitación de los agricultores y la organización de sus comunidades para que utilicen 
racionalmente los recursos productivos que realmente poseen, y para que ellos mismos puedan protagonizar la solución de sus problemas, con menor dependencia de los factores externos y escasos recursos a lo que nos hemos referido.

Para enfrentar todos estos nuevos y poco conocidos desafíos, es indispensable la formación de los técnicos agropecuarios a las circunstancias de cada región del país, sólo así ellos, estarán en condiciones de ayudar a mejorar, las cada vez mayores necesidades de los agricultores.

Las soluciones que se deben plantear y entrar en corrección no están en la solución política de los eventos que se causen en el campo y para reflexionar un poco sobre este tema se trae a colación el pensamiento de Nikolai Bukharin, economista y político soviético (1888-1938), quien desarrolla el pensamiento de que: En la economía las soluciones rinden más que los problemas; en la política, los problemas valen mucho más que las soluciones.

Haciendo un recorrido por una serie de publicaciones a nivel nacional e internacional de las problemáticas que afectan el sector rural, hemos encontrado que se han hecho múltiples estudios y trabajos para identificar las infinidad de causas de la pobreza y el subdesarrollo rural y finalmente la mayoría de ellos ha llegado a la conclusión que la causa principal es la insuficiencia de conocimientos por parte de la gente del campo.

Los proyectos de desarrollo rural implementados por los gobiernos, o por cualquier ente ocupado en el desarrollo agrícola, solo tendrán éxito después que proporcionen a los afectados por el subdesarrollo, en primer lugar, el saber y el saber hacer para que puedan actuar como constructores del desarrollo, es decir proyectos acompañados de capacitación no solamente en la ejecución del proyecto, si no también en su comercialización, en darle valor agregado a su producción y en mejorar la competitividad del sector al que están dedicados.

Existe un creciente consenso de que la agricultura, en su globalidad, sólo conseguirá enfrentar con éxito sus antiguos y especialmente sus nuevos desafíos, si los agricultores adquieren nuevos conocimientos, habilidades y destrezas, e incluso si adoptan actitudes diferentes. Si no lo hacen, difícilmente podrán realizar una agricultura eficiente y sin ella no tendrán rentabilidad ni competitividad.

"Hemos estado acostumbrados a pensar en el capital como el factor escaso en
la producción y en su transferencia como el instrumento clave para el
crecimiento. El conocimiento es ahora tan, si no más, importante factor de
desarrollo, y esta situación tiende a intensificarse. En el próximo siglo la
acumulación y aplicación del conocimiento conducirán los procesos de
desarrollo y crearán oportunidades, sin precedentes para el crecimiento y la 


\section{reducción de la pobreza. Sin embargo, existen riesgos significativos para incrementar la desigualdad entre y dentro de las naciones." James D Wolfensohn, Presidente del Banco Mundial, 17 de marzo 1997.}

Cabe destacar que una de las causas de la pobreza rural generalmente no es porque no hay recursos productivos, sino que se da por falta de conocimientos, actitudes, aptitudes y valores, con los cuales el productor agropecuario puede auto corregir sus propias ineficiencias y así utilizar los recursos disponibles con mayor eficiencia y productividad. Uno de los factores que incide son los sitios donde se presta la educación, ya sean escuelas rurales, escuelas técnicas o de facultades de ciencias agrarias con currículos obsoletos y que no están formando sus estudiantes debidamente para la capacitación empresarial, para enfrentar los retos de la agricultura moderna, sin olvidar las pequeñas granjas de productores, que han sido abandonados por el Estado, en cuanto a programas de asistencia técnica y que prácticamente están en quiebra, siendo abandonadas por sus dueños con un éxodo masivo a las capitales de departamentos en algunos puntos de la geografía colombiana.

Para corregir esto no es necesario empezar desde arriba (Ministerio de educación, rectores de universidades, decanos de facultades agropecuarias y de educación, etc.), sino desde abajo. La corrección de estas ineficiencias debe comenzar, por el establecimiento de proyectos de extensión con extensionistas bien capacitados en todos los procesos de desarrollo del campo, profesores con conocimientos mas profundos en estos procesos, tanto de las facultades de ciencias agrarias como de economía, con programas de economía agrícola y administración agropecuaria, visionando el desarrollo desde adentro (de las facultades), hacia fuera, para el campo, para un mayor desarrollo del país, donde se establece entonces que el conocimiento es el factor mas importante del desarrollo rural. Donde se podrá ponderar la riqueza de un país por la riqueza de sus productores agropecuarios.

En el momento que un productor agropecuario sea capaz de sostener a su propio agrónomo, medico veterinario o especialista agrícola, es cuando evidenciamos su riqueza no solo en el aspecto económico sino también en sus conocimientos, ya que aprenderá del manejo de su finca, de su hato ganadero y de como administrar bien sus recursos.

Actualmente ser eficiente ya no es una ventaja sino un requisito y sólo van a sobrevivir económicamente los agricultores que sean altamente eficientes en los aspectos técnicos, gerenciales y organizativos de las distintas etapas del negocio agrícola, lo quiere decir que la eficiencia dejó de ser una ventaja competitiva para transformarse en un requisito para poder sobrevivir en la actividad agrícola y para alcanzar esta eficiencia hay que hacerla con menos crédito, menos subsidios y menos 
protección, pero solamente con campesinos mejor capacitados, que les permitirá ser más productivos en el negocio agrícola, y algo muy esencial: Que trabajen asociados. Aplicar este factor les daría mas independencia económica en todos los aspectos.

Esto significa que los escasos insumos materiales tendrán que ser potenciados a través de una correcta utilización grupal. Se debe hacer un cambio total en el concepto de uso de aquellas máquinas y equipos que "cuestan mucho y se utilizan poco", a efectuarlas para un desarrollo colectivo que permita disminuir gradualmente costos operativos de estas, logrando con ello disminuir los costos de producción en general en la producción agropecuaria y la agregación de valor, lo que les permitirá ser mas rentable.

El éxito de los agricultores y de los trabajadores rurales llegara a aquellos que estén más capacitados y organizados con propósitos empresariales y esto les va a permitir mayores rendimientos por hectárea y por unidad animal, eliminar el sobredimensionamiento y ociosidad, reducir costos de producción, mejorar la calidad de sus productos y agregar valor a sus cosechas practicando la transformación de productos en procesos agroindustriales así sea artesanales, reduciendo considerablemente los eslabones de intermediación, tanto en la adquisición de insumos como en la comercialización de sus excedentes.

Los agricultores bien capacitados deben eliminar sus propias ineficiencias para aumentar sus actuales rendimientos, los cuales son muy bajos y no son competitivos frente a los grandes productores a nivel mundial. El agricultor asociado debe trabajar con mayor eficiencia y multiplicar sus conocimientos para realizar nuevas actividades ejecutadas actualmente con una eficiencia y productividad muy baja y que en el resto del mundo se ejecutan a un nivel muy alto por los competidores agrícolas

Solucionar el problema de la deficiencia educacional por una educación emancipadora, permitirá conciliar escasez de recursos con eficiencia empresarial. Algunos ejemplos hipotéticos ilustran la factibilidad y eficacia de un modelo más endógeno y emancipador, y un ejemplo seria pasar de sembrar un monocultivo que produce alimentos e ingresos una o dos veces al año,(como el caso de la producción cafetera, cacaotera y arrocera), y comenzar a implementar una gradual diversificación agrícola-ganadera con el propósito de generar ingresos y alimentos, tanto para la familia como para los animales durante los 365 días del año, y un modelo podría ser una granja integral, que nos permitiría utilizar todos los desechos de la granja (estiércoles, subproductos de las cosechas, etc.), pasando a convertirlas en abonos orgánicos que nos permitirán disminuir los costos de producción en las fincas. Al sembrar productos mas diversificados nos permitirá obtener mas cosechas de 
diferentes rubros agrícolas y pecuarios y así mejorar los ingresos de cada productor agropecuario.

Diversificar la producción, automáticamente reduce la crónica dependencia del crédito y disminuye riesgos sanitarios, climáticos y comerciales. Polan Lacki, ex asesor de la FAO para América Latina nos da algunos ejemplos sobre esta temática. En vez de adquirir y mantener un toro y cinco vacas genéticamente mediocres y subalimentadas que rinden en total apenas 20 litros de leche al día y cada una de ellas tiene un parto cada 22 meses, será preferible deshacerse del semental( quien sale costoso por su alimentación y cuidados), y de cuatro hembras, porque suelen consumir más de lo que producen. Con el dinero obtenido, mejorar la producción de forrajes y adquirir una o dos vacas genéticamente mejoradas, las cuales bien alimentadas podrán producir hasta dos veces diarios la cantidad de leche, y un ternero a cada 12 meses y con ello logramos aumentar la productividad del predio casi al mismo costo.

Así mismo en el área agrícola: En vez de sembrar una hectárea de papa, con graves errores tecnológicos e insuficiencia de insumos que rinde apenas 10 toneladas, será preferible corregir dichos errores, ahorrar trabajo innecesario y concentrar los escasos insumos disponibles, tal vez en un tercio de hectárea y en ésta menor superficie cosechar las mismas 10 toneladas, o implementar la siembra de variedades más productivas, que nos genere mayor producción por hectárea y de esta forma reducir el hectariaje de siembra e introducir otros cultivos, así mismo altamente productivos y ampliando la diversificación de la producción. Al reemplazar la cantidad por la calidad y aumentando la productividad, los agricultores disminuirán las inversiones y ociosidades de la tierra, trabajaran menos y ganaran más y en forma mas permanente, al implementar la diversificación de la producción en sus predios.

Lo mas grave que esta ocurriendo en Colombia y en muchos países de la región es que la gran mayoría de productores agropecuarios no están asociados en ninguna agremiación. Al no estar asociados, generalmente compran el alimento balanceado para la cría de sus animales (cerdos, vacas, pollos, etc.), con alto valor agregado al último eslabón de intermediación( almacenes agropecuarios), y posteriormente venden los animales, sin valor agregado (vivos ), al primer eslabón de la cadena, cuando les sería más rentable producir gran parte de los alimentos en la propia finca diversificada, y comercializar los animales, proporcionándoles mayor valor agregado es decir ya sacrificados, en canal y fríos, aprovechando muchas de las partes internas de los animales, utilizando sus pieles y otros subproductos de estos y vendiéndole al último eslabón de la cadena, que es donde se obtiene mayor ganancia. 
En la medida de lo posible, la finca tiene que ser transformada en una agroindustria familiar productora de algunos insumos, como por ejemplo bloques multinutricionales y semillas de variedades de la región, seleccionando las de mejor rendimiento, producir abonos orgánicos (utilización de los estiércoles para la producción de bioabono), producción de forrajes con alto valor nutritivo, que le incorpore mas valor a los excedentes que se producen en ella. Con solo lograrlo se tendrá una menor cantidad de intermediarios, menos impuestos, menos fletes y menos peajes.

La solución la encontramos a la mano y son muy sencillas, eficaces y de bajo costo. Es aquí donde entra a jugar un papel incidente la Universidad. La unificación de propósitos de investigación de las dos Facultades, sus institutos de investigación en el área agrícola y pecuaria y el de las ciencias económicas y empresariales. Formar empresarios del campo, así sea con los pocos recursos que poseen y tecnificar el campo con proyectos de diversificación, que les permitan ser mas eficientes en el negocio agrícola.

Para ello debemos efectuar una diversificación productiva, implementándola con una gradualidad tecnológica, con el propósito de que los recursos necesarios para financiar la intensificación productiva sean auto-generados en las propias fincas y esto implica mejorar la organización de la producción en todos los ámbitos para eliminar sobredimensionamientos y ociosidades y verticalizar el negocio agrícola.

Un factor muy importante, y que cambia casi que radicalmente el negocio agrícola, es la implementación de una agricultura por contrato. Al estar asociados los productores agropecuarios pueden establecer en su asociación contratos de producción con determinadas empresas agroindustriales, permitiéndoles ir a la fija en su producción, ya que tienen el cliente final quien garantizaría la compra de sus productos. Esto al mismo tiempo les hará producir con mejor calidad ya que deben conservar a su cliente final.

En el aspecto educativo la escuela rural debe enseñar la aplicación de posibles soluciones a toda esta problemática. Para la mayoría de las familias rurales el paso por la escuela básica rural en nuestro caso (Colombia hasta último grado bachiller), es la única oportunidad en sus vidas de adquirir las competencias que les permitirían eliminar las principales causas internas del subdesarrollo rural. Sin embargo, muchas por no decir la gran mayoría de estas escuelas no están cumpliendo con esta importantísima función emancipadora de dependencias y de vulnerabilidades; porque sus contenidos y métodos son disfuncionales, inadecuados y obsoletos y no están al día con las necesidades productivas y familiares del mundo rural. 
Las escuelas fundamentales rurales serían mucho más útiles si, antes de enseñar la historia de Europa o la geografía de Asia, enseñasen a sus alumnos la historia y la geografía de sus comunidades. Si, en vez de distraer las atenciones de los educandos con las jirafas y elefantes de África, les enseñasen como criar, con mayor eficiencia, los animales existentes en sus fincas con la finalidad de mejorar el auto-abastecimiento y el ingreso familiar.

Dichas escuelas serían más útiles si enseñasen a los niños cómo evitar las plagas de la agricultura y de la ganadería, cómo identificar y eliminar las plantas que intoxican sus animales y los insectos que transmiten las enfermedades, enseñándoles todo lo concerniente a la alelopatía, lo que disminuiría el uso y por ende disminuirías los costos en agro tóxicos, contaminantes del medio ambiente en vez de hacerles memorizar la longitud del Río Nilo, sería más útil enseñarles cómo y porqué deberían evitar la polución de otro río: como por ejemplo el río Pamplonita, y otros ríos de nuestro departamento, como mejorar sus afluentes, así mismo disminuyendo la deforestación, implementando la siembra de guadua y de otras especies que nos permitirán recuperar los caudales de nuestros ríos..

Sería conveniente enseñarles cómo y porqué deberían plantar huertas y árboles frutales diversificados en sus fincas y cómo adoptar medidas de conservación del suelo para que éste siga produciendo con altos rendimientos. Mejorar en el aspecto educativo sobre uso de alimentos sanos, proporcionadamente balanceados en cuanto a calorías, vitaminas y minerales y dirigir la producción de las diferentes regiones al uso de estos alimentos y con ello al mismo tiempo capacitaríamos la población rural para la producción diversificada de alimentos en sus propios predios, lo que disminuiría considerablemente los índices de desnutrición en el campo.

Algo que se debe implementar es la enseñanza sobre la propia comunidad rural en que se habita, el aspecto de la producción agropecuaria, de la enseñanza en el saber hacer de cada comunidad para que estos ejemplos sean aplicados por la comunidad, la región y el país.

En dichas escuelas se aburre a los niños exigiéndoles que memoricen temas de escasa y dudosa relevancia y no se les enseña en forma creativa, participativa y práctica lo que sí necesitan aprender para creer mas en si mismos, ser más emprendedores, más autogestionarios y más independientes. De esas escuelas siguen egresando generaciones de futuros agricultores, agricultoras, padres y madres de familia, con bajísima autoestima, sin los conocimientos, sin las actitudes y sin los valores que necesitan para ser agricultores más eficientes, mejores educadores de sus hijos y solidarios protagonistas de sus comunidades. 
Las escuelas deben formar solucionadores de problemas. Nos hemos olvidado de algo tan importante como el conocimiento como el factor primordial para el desarrollo del campo y se le ha dado mas relevancia al capital como el factor escaso en la producción y en su transferencia como el instrumento clave para el crecimiento, sin tener en cuenta que sin conocimiento no podemos hacer buen uso de este.

Quienes dirigen la agricultura latinoamericana están mas dedicados a la política que a la economía, cuando debe ser lo contrario. Casi a todo le dan seudo soluciones que confunden aún mas a los agricultores. El sector agrario necesita soluciones de verdad, más perdurables en el tiempo, que sean más reales y que se cumplan eliminando causas tan profundas como la rentabilidad y competitividad tan baja y no prometer subsidios que no van a entregary que generalmente no van a cumplir. En otras palabras debemos salir adelante por nuestra cuenta sin tener que recibir algo del gobierno, sacándonos de encima ese paternalismo dependiente, pero resaltando que sin educación para mejorar los índices de producción y rentabilidad nunca saldremos adelante en el desarrollo agropecuario.

Promover e implementar proyectos de capacitación para mejorar la productividad $\mathrm{y}$ por ende la rentabilidad del sector agropecuario y del incipiente sector agroindustrial protagonizado por comunidades de bajos recursos económicos debe ser prioridad de las universidades. En ello deben trabajar las facultades antes mencionada y también la Facultad de alimentos y medio ambiente.

Un aspecto fundamental e importante es el caso de las mujeres campesinas. Ellas representan en la población rural más de la mitad del sector. A esta población se le debe dar mas oportunidad de opinión y aporte de ideas en el trabajo del campo ya que generan un buen porcentaje de ingresos dentro de la producción agropecuaria. En el país tenemos ejemplos que deben ser difundidos, como en el caso de los productores de feijoa en Tibasosa, cerca a Bogotá, en el que la mujer es un factor muy importante en el desarrollo de esta fruta, donde le dan un valor agregado bastante fuerte a este producto, produciendo vinos, mermeladas, conservas, etc. Trabajo prácticamente efectuado por las mujeres de este municipio dedicadas al sector agrícola.

En América latina existe un evidente creciente desequilibrio entre las múltiples y urgentes necesidades de los agricultores y las decrecientes de los debilitados y endeudados gobiernos en satisfacerlas y como si fuera poco los escasos recursos que el gobierno destina al agro se vuelven insuficientes por la forma como son asignados. Actualmente tenemos el caso de Argentina, con huelgas del sector agropecuario y la debacle en que se encuentra el Estado venezolano con la producción de alimentos. 
Ante este creciente desbalance que existe entre el gobierno y la población rural no se pueden seguir formulando agotadas propuestas paternalistas por mejores que sean las intenciones de donde provengan, esta es una actitud que causa más daño que beneficios a los agricultores porque los desorienta y estimula su pasividad. Generalmente son engañados esperando por recursos y decisiones que los gobiernos no pueden proporcionarles, ya estas propuestas llegaron a su límite y no se puede seguir prometiendo. La propuesta de una agricultura con un desarrollo endógeno permitirá paliar la crisis, pero esta propuesta debe estar acompañada de capacitación permanente hasta lograr productores agropecuarios mas preparados para enfrentar los desafíos actuales de la globalización y de la era del biocombustible.

La solución entonces es un cambio de propuesta y esta debe ser una educación emancipadora. Los gobiernos no están en condiciones de realizar todo por los agricultores y tendrán que asumir el papel de emancipador en el aspecto educador las Universidades. No se debe estimular la dependencia del productor, el propósito de todos los proyectos agrícolas es que los propios agricultores solucionen la mayor cantidad de problemas que agobian su existencia y encontrar ellos mismos gran parte de la solución de estos, en vez de alimentar en ellos la nueva ilusión de que el mercado y las cadenas agro-alimentarías lo harán por los productores; porque el mercado y el agribusiness están preocupados en resolver sus propios problemas, y no necesariamente los de los productores rurales.

Para preparar esta progresiva emancipación, los gobiernos deberán promover formas sencillas de organización empresarial de los agricultores y proporcionarles los conocimientos mínimos que ellos necesitan para que verdaderamente quieran, sepan y puedan asumir, en forma gradual, actitudes y roles más protagónicos en la eficiente solución de sus propios problemas pasando de una bajísima productividad y rendimiento de todos los factores de producción que ellos ya poseen, con el elemental propósito de que cada unidad de mano de obra, tierra, insumo, crédito, animal, o tractor, por el simple hecho de que es escasa, cara o insuficiente produzca con más eficiencia una mayor cantidad de granos, tubérculos, frutas, hortalizas, forraje, carne, lana o leche; y especialmente de ingresos, con una diversificación de la producción mas amplia, mucho mas eficiente y que si le da ingresos durante todo el año y no solamente en épocas de cosechas distanciadas y que generalmente lo mantienen endeudado.

El Gobierno debe y es una condición sine qua non, proporcionar a los agricultores las competencias para que ellos puedan producir más y mejor con menos recursos, con mínima participación del Estado. Esto seria lo mas importante para lograr el éxito del campo colombiano. 
Muchas veces el subsidio que da el gobierno (en algunos países de la región), es un premio a la ineficiencia. Esto se elimina con mayores posibilidades de conocimientos. Muchas de la ineficiencias son provocadas generalmente por errores primarios corregibles con conocimientos elementales y no tanto de políticas generosas ni de créditos abundantes. Para confirmarlo basta con analizar, sin eufemismos: los bajísimos índices y rendimientos promedio de la agricultura y de la

ganadería latinoamericana, los errores primarios que ocurren en la aplicación de las tecnologías y en el uso de los recursos disponibles y finalmente las gravísimas distorsiones en la forma como los productores rurales adquieren los insumos y cómo comercializan sus excedentes.

Es evidente que estas ineficiencias no ocurren por culpa de los agricultores. Ellas son provocadas por la profunda disfuncionalidad de la educación formal rural en los tres niveles y por el lamentable debilitamiento de la extensión agrícola. Es por este motivo que la profunda transformación de las escuelas básicas rurales, es un pre-requisito que deberá ser potenciado o sinergizado con idénticas adecuaciones en las facultades de ciencias agrarias, en las escuelas agrotécnicas y en los servicios de extensión rural, públicos y privados. Todas estas instituciones deberán pasar por una reingeniería en los contenidos educativos y en los de métodos pedagógicos, los que ojala prioricen el "enseñar a solucionar los problemas solucionándolos"; en fin por una reingeniería de calidad educativa.

Sin embargo, educación de calidad no necesariamente significa construir más edificios, adquirir más computadoras, instalar laboratorios sofisticados u ofrecer más cursos en el exterior. Lo que sí se necesita es tener el coraje de "poner el dedo en la llaga", reconocer sin eufemismos y enfrentar con determinación el problema de fondo. Es decir, el profundo desencuentro entre el qué y cómo se enseña en las escuelas y el qué y cómo las familias rurales realmente necesitan aprender, este divorcio es inaceptable y es por esta razón adicional que los líderes más lúcidos del agro latinoamericano están exigiendo una revolución educativa, de realismo, de objetividad y de pragmatismo, que permita formar una nueva generación de mujeres y hombres rurales que quieran, sepan y puedan protagonizar la revolución productiva de la eficiencia y de la emancipación.

En resumen, la educación es el principal problema que tiene la población rural ya que la mayoría no la poseen y si la poseen, esta no es de muy buena calidad, por lo que hay que hacer es un cambio total en el sistema educativo rural desde arriba, derribando los muros interpuestos tanto mentales como materiales

El gobierno es el gran culpable ya que promete demasiado y no cumple, y si llega a cumplir lo hace a medias delegando funciones a funcionarios corruptos, a maestros incompetentes, sin visión del agro, que cumplen sus horarios por cumplir, por 
compromisos políticos.( caso del Chocó, donde la corrupción campea desde tiempos inmemoriales, en todos los aspectos).

Ya en el contexto del problema Colombiano hay que agregar que la problemática del desalojo por grupos de extrema derecha o de izquierda ahonda mas aun la crisis a la que esta sometido el campesinado. Reconociendo que la problemática más grave sigue siendo aún la siembra de coca en muchas regiones del país yel incremento del área sembrada.

El gobierno nacional debe implementar medidas aún mas radicales en cuanto a la devolución de tierras en los sitios en que fueron desalojados los campesinos, asegurar el área implementando cultivos comerciales y de alimentos para garantizar la seguridad alimentaria de estas regiones empobrecidas por la guerra que se desarrolló en ellas.

\section{CONCLUSIONES}

El campo latinoamericano en estos momentos esta pasando por una crisis económica abismal; debido a las inadecuadas medidas implementas por los gobiernos que en vez de preocuparse por el conocimiento de los habitantes del campo adoptan medidas económicas como subsidios o prestamos entre otros, como parte de una estrategia para solucionar los problemas que atraviesa el sector rural; pero cuyas medidas no solucionan de una forma radical los problemas, sino que son medidas parciales de corto periodo de tiempo y que no tiene ninguna repercusión.

Una de las causas del empobrecimiento del campo radica en la educación impartida en las escuelas rurales cuyos contenidos programáticos no se ajustan a la realidad del campo y que pueden ser la clave de la solución de los problemas actuales; es decir se debe optar por una educación en donde la prioridad sea el ofrecer un conocimiento útil y aplicable a la realidad del sector rural.

Para poder enfrentar los retos del siglo XXI y solucionar la problemática actual, los agricultores de América Latina deben emplear el asociativismo para lograr ser eficientes, reducir costos y tener una economía en gran escala, en este sentido se podrá salir del subdesarrollo en el cual están.

Uno de los objetivos del siglo XXI a lo cual los gobiernos de Latinoamérica se comprometieron es el de erradicar el hambre, para conseguirlo es necesario dirigir toda nuestra atención hacia el campo y solucionar los problemas que están frenando su desarrollo. 
Tenemos entonces que el campo se caracteriza por una economía inestable con efecto en competitividad, Problemas de paz y convivencia social, escasez de mano de obra por la migración a las ciudades. Los acelerantes para el desarrollo deben ser: Mayor desarrollo de una economía solidaria y el fomento en general del sentido de solidaridad, con mayor participación y poder comunitario.

El desarrollo del campo debe estar basado en planes y proyectos acompañados con dirección técnica y científica, con una producción diversificada y sostenibilidad al medio ambiente, con una producción limpia. El componente básico de este proceso es aumentar el nivel educativo del productor agropecuario acompañándolo de tecnología especializada y con disponibilidad de recursos tecnológicos.

Un deber primordial de las universidades es formar profesionales agropecuarios con capacidades empresariales, que impulsen el desarrollo de la agroindustria, que estimulen la conformación de unidades productivas más grandes y eficientes y que se establezcan programas de extensión especializada y regionalizada.

\section{REFERENCIAS}

- www.zoetecnocampo.com/foro/Forum1/HTML/000034.html

- www.polanlacki.com.br/agroesp 